\title{
The Alternative to Laparoendoscopic Single-Site Surgery: Small Strategic Laparoscopic Incision Placement (SLIP) Nephrectomy Improves Cosmesis Without Technical Restrictions
}

\author{
Nina Casanova, M.D., and J. Stuart Wolf, Jr., M.D.
}

\begin{abstract}
Background: The latest attempt to improve the cosmesis of laparoscopic surgery is laparoendoscopic single-site surgery (LESS). We present our initial experience with an alternative procedure with similar cosmetic benefit but without technical limitations.

Methods: Small strategic laparoscopic incision placement (SLIP) nephrectomy is performed transperitoneally, generally using three 5 -mm ports (one in the umbilicus) and one 12-mm port placed below the pubic hairline, such that only two 5-mm scars are visible without close inspection. We assessed our first 21 procedures, which included all but five of the standard transperitoneal nephrectomies by a single surgeon from June 2008 through July 2009. These were matched 1:2 (exactly by gender and American Society of Anesthesiology score, and then closest in age and body mass index) from 96 patients undergoing similar standard transperitoneal laparoscopic procedures from 2005 through 2008.

Results: The SLIP and control groups were well matched, with mean age and body mass index differing by only 3.6 years and 1.1, respectively. Of the SLIP patients, $34 \%$ were obese or morbidly obese, and a trainee was the primary surgeon in $81 \%$ of cases. Mean operative time was 23 minutes longer in the SLIP cases. There was no difference between groups in estimated blood loss, complication rate, or convalescence.

Conclusions: Like LESS, SLIP nephrectomy provides improved cosmesis. Unlike LESS, it is only slightly more difficult to perform than standard laparoscopic nephrectomy and can be performed in technically challenging cases (obesity, large specimen, etc). Similar to the literature on LESS, there is no convalescence benefit to SLIP nephrectomy; the advantage over standard laparoscopy is purely cosmetic.
\end{abstract}

\section{Introduction}

S INCE THE FIRST laparoscopic nephrectomy there has been a relentless effort to decrease invasiveness and improve cosmesis associated with renal surgery. Most recently, this is evidenced by interest in laparoendoscopic single-site surgery (LESS), which is purported to decrease morbidity and improve cosmesis while maintaining oncological principles. ${ }^{1}$ Although this may be an exciting new horizon for some surgeons, the technical difficulty of the approach poses a hurdle to widespread use by the practicing urologist. Moreover, most evidence suggests that the benefit of LESS over standard laparoscopy is only cosmetic, with no improvement in convalescence measures. $^{2-10}$ In this article we present our small strategic laparoscopic incision placement (SLIP) technique as an alternative to traditional laparoscopy, which provides cosmetic benefits similar to LESS but without the technical limitations.

\section{Methods \\ SLIP technique}

The SLIP nephrectomy is similar in its surgical progression to the standard transperitoneal laparoscopic nephrectomy. Patients are placed in a $45^{\circ}$ lateral decubitus position. Generally three 5-mm ports are inserted, including the port for the 5 -mm $30^{\circ}$ to $45^{\circ}$ video laparoscope placed within the umbilicus plus two ports placed superior and lateral to the umbilicus. A fourth 5-mm port is placed for liver retraction, if needed. The only larger port is a $12-\mathrm{mm}$ one, inserted below the pubic hairline. This port is used for larger instruments, stapler insertion, and specimen extraction. As such, there generally are only two (or three if liver retraction is needed) 5 -mm incisions visible postoperatively without close inspection (Fig. 1). If necessary, extra-long staplers and other instruments (as for bariatric surgery) can be used through the

Department of Urology, University of Michigan Health System, Ann Arbor, Michigan. 


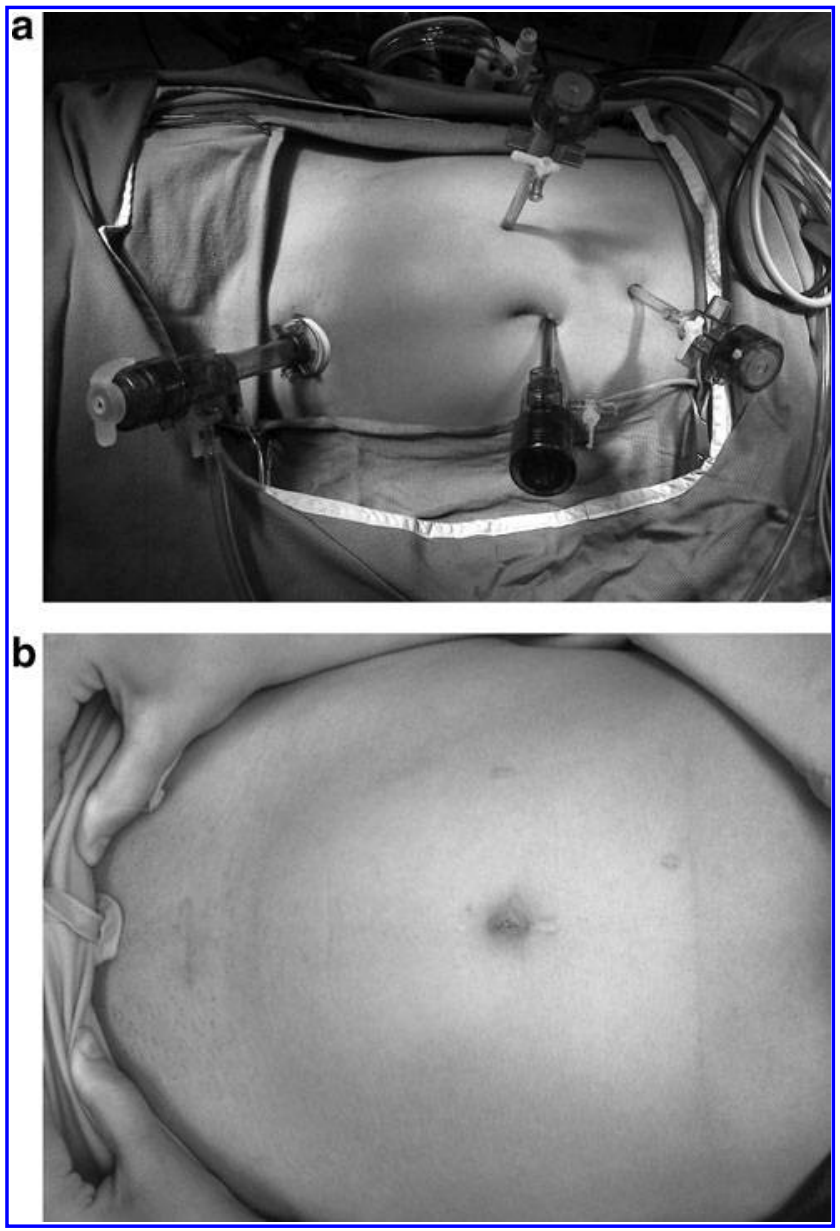

FIG. 1. (a) Port placement for right simple nephrectomy with SLIP technique, including 5-mm laparoscope port (in this case at upper edge of umbilicus after excising scar from prior umbilical jewelry), two 5-mm working ports, and a 12-mm assisting port (used for instruments larger than $5 \mathrm{~mm}$, and for specimen extraction) below the pubic hairline. (b) Abdominal scars in the same patient 6 months later. $\mathrm{SLIP}=$ small strategic laparoscopic incision placement.

12-mm suprapubic site (Fig. 2), which, although distant from the site of surgery, provides acceptable angle of approach to the operative site. The specimen is removed via morcellation through the 12-mm port site, which is enlarged transversely to 3 to $4 \mathrm{~cm}$ for morcellation under vision in cases of malignancy but is left small for morcellation of benign specimens. ${ }^{11} \mathrm{We}$ routinely inject all port sites with $0.5 \%$ bupivacaine at the start of all laparoscopic procedures.

\section{Data collection and analysis}

We identified retrospectively the first 21 consecutive laparoscopic nephrectomies performed by a single surgeon (J.S.W.) using the SLIP technique, including radical nephrectomy $(n=10)$, simple nephrectomy $(n=8)$, and heminephrectomy for benign disease $(n=3)$. All cases were carried out from June 2008 to July 2009. This group included all but five of the standard transperitoneal radical, simple, and heminephrectomies by J.S.W. from June 2008 through July 2009. SLIP nephrectomy was not done in five patients because of

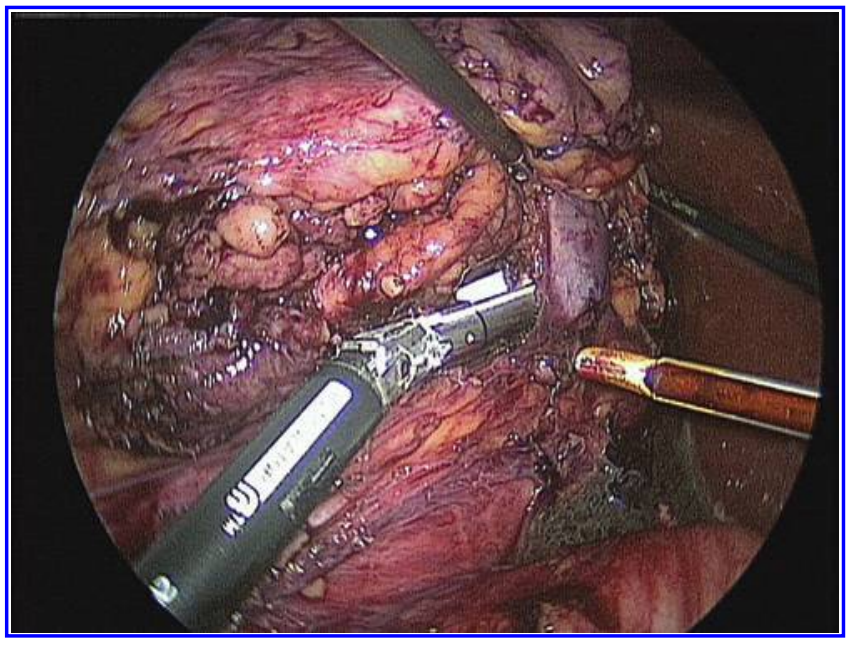

FIG. 2. Intraoperative view of right SLIP radical nephrectomy in patient with a body mass index of $44 \mathrm{~kg} / \mathrm{m}^{2}$ through the $5-\mathrm{mm}$ umbilical port. The operative field is surrounded by instruments coming from different directions. An extralong stapler is used through the $12-\mathrm{mm}$ suprapubic port. Working instruments enter through 5-mm ports from the left and the right. In this case a 5 th port $(5-\mathrm{mm})$ was necessary for a liver retractor.

locally advanced renal cancer $(n=1)$, anticipated peri-renal inflammation $(n=1)$, and prior full midline incision $(n=3)$.

The 21 SLIP nephrectomy patients were compared 1:2 with matched control cases selected from 96 standard (non-handassisted) transperitoneal laparoscopic radical, simple, and hemi-nephrectomies performed from June 2005 to July 2008. The SLIP and control patients were matched exactly by gender and American Society of Anesthesiology score, and then as closely as possible by age and body mass index (BMI). We compared demographics, operative factors, and outcomes. Data were collected prospectively using our IRB-approved database, and also retrospectively through chart review. The length of the extraction site incision was measured at the conclusion of the procedures. Parenteral morphine sulfate equivalents were calculated as $\mathrm{mg}$. morphine sulfate plus $\mathrm{mg}$. meperidine divided by 10 plus $\mathrm{mg}$. hydromorphone times 10 plus mg. ketorolac divided by six. Statistical analyses were performed using commercial software. $p$-values $\leq 0.05$ were considered significant. For comparing variables between patient groups, we used the chi-squared or Fisher Exact test for categorical variables and the Mann-Whitney $U$ test for continuous variables.

\section{Results}

The SLIP and control groups were well matched, with exact matching by age and American Society of Anesthesiology scores, and with mean age and BMI differing by only 3.6 years and $1.1 \mathrm{~kg} / \mathrm{m}^{2}$, respectively (Table 1). Notably, of the SLIP patients, $24 \%$ had BMI 30 to $40 \mathrm{~kg} / \mathrm{m}^{2}$ (obese) (Fig. 3) and $10 \%$ had BMI $>40 \mathrm{~kg} / \mathrm{m}^{2}$ (morbidly obese), which was similar to the control group.

There was no difference in terms of prior abdominal surgery and distribution of procedure types between the groups (Table 1). The primary operator was a trainee in the majority of cases from either group (87\% overall), and there was no 
Table 1. Comparison of Small Strategic Laparoscopic Incision Placement (SLIP) and Control Group

\begin{tabular}{|c|c|c|c|}
\hline Demographic factor & SLIP group $(\mathrm{n}=21)$ & Control group $(\mathrm{n}=42)$ & $\mathrm{p}$-Value \\
\hline Age-mean (range) & $50(24-84)$ & $53(26-79)$ & 0.23 \\
\hline \multicolumn{4}{|l|}{ Gender } \\
\hline No. of men (\%) & $8(38 \%)$ & $16(38 \%)$ & \multirow[t]{2}{*}{$>0.99$} \\
\hline No. of women (\%) & $13(62 \%)$ & $26(62 \%)$ & \\
\hline \multicolumn{4}{|l|}{ ASA score } \\
\hline No. of ASA 1 (\%) & $3(14 \%)$ & $6(14 \%)$ & \multirow[t]{3}{*}{$>0.99$} \\
\hline No. of ASA $2(\%)$ & $10(48 \%)$ & $20(48 \%)$ & \\
\hline No. of ASA $3(\%)$ & $8(38 \%)$ & $16(38 \%)$ & \\
\hline BMI, $\mathrm{kg} / \mathrm{m}^{2}$-mean (range) & $29.4(20.1-48.9)$ & $29.8(20.1-48.9)$ & \multirow[t]{4}{*}{0.38} \\
\hline Nonobese $<30$ (No. of patients [\%]) & $14(67 \%)$ & $29(69 \%)$ & \\
\hline Obese 30-40 (No. of patients [\%]) & $5(24 \%)$ & $9(21 \%)$ & \\
\hline Morbidly obese (No. of patients [\%]) & $2(10 \%)$ & $4(10 \%)$ & \\
\hline No. of patients with prior abdominal surgery $(\%)$ & $4(19 \%)$ & $16(38 \%)$ & 0.16 \\
\hline \multicolumn{4}{|l|}{ Procedures } \\
\hline No. of radical nephrectomy (\%) & $10(48 \%)$ & $28(67 \%)$ & \multirow[t]{3}{*}{0.12} \\
\hline No. of simple nephrectomy (\%) & $8(38 \%)$ & $13(31 \%)$ & \\
\hline No. of hemi-nephrectomy (\%) & $3(14 \%)$ & $1(2.4 \%)$ & \\
\hline \multicolumn{4}{|l|}{ Operative outcomes } \\
\hline Operative time, minutes-mean (range) & $187(108-265)$ & $164(100-288)$ & 0.02 \\
\hline $\mathrm{EBL}, \mathrm{mL}$-mean (range) & $185(25-750)$ & $150(25-600)$ & 0.51 \\
\hline \multicolumn{4}{|l|}{ Primary operator } \\
\hline No. of cases trainee $(\%)$ & $17(81 \%)$ & $38(90 \%)$ & \multirow[t]{2}{*}{0.42} \\
\hline No. of cases attending (\%) & $4(19 \%)$ & $4(10 \%)$ & \\
\hline \multicolumn{4}{|l|}{ Convalescence measures and complications } \\
\hline No. of patients with Clavien grade $1-2$ complication (\%) & $7(33 \%)$ & $9(21 \%)$ & 0.36 \\
\hline No. of patients with Clavien grade $3-5$ complication (\%) & $1(4.8 \%)$ & $4(9.5 \%)$ & 0.66 \\
\hline $\mathrm{MSO}_{4}, \mathrm{mg}$ equivalents-mean (range) & $34(3-81)$ & $26(2-82)$ & 0.18 \\
\hline LOS, days-mean (range) & $1.6(1-3)$ & $2.1(1-8)$ & 0.42 \\
\hline
\end{tabular}

$\mathrm{ASA}=$ American Society of Anesthesiologists; $\mathrm{BMI}=$ body mass index; SLIP = small strategic laparoscopic incision placement; $\mathrm{LOS}=$ length of stay .

difference between the groups. Mean operative time was 23 minutes longer in the SLIP cases $(p=0.02)$, but all other operative parameters were similar.

Complications were categorized using the modified Clavien system. No significant intraoperative complications or conversions to open surgery were noted in either group. Postoperatively, 33\% of the SLIP group versus $21 \%$ of the control group had a Clavien category 1 or 2 complication, and $4.8 \%$ versus $9.5 \%$, respectively, had a complication of Clavien category 3 or greater. None of these rates was significantly different.

As would be expected, there was considerable variability in the number and size of ports used, as well as in the locations of port placement. A mean of 3.4 5-mm ports was used in the SLIP group versus 2.3 in the control group. Only one 12-mm port was used in the former group compared with 1.9 in the latter group ( $p<0.0001$ for both comparisons). The placement of the extraction-site incision differed between the groups, with all but three of the extraction incisions in the SLIP group being placed in the transverse suprapubic location described above (all three nonsuprapubic incisions were made in lower midline scars from prior operations), compared with $73 \%$ of the control group extraction-site incisions being in subcostal, paramedian, or flank locations $(p<0.0001)$. Despite these differences, the mean total incision length of the 2 groups was comparable at $3.3 \mathrm{~cm}$ (SLIP group) and $3.8 \mathrm{~cm}$ (control group) $(p=0.36)$.

Finally, short-term convalescence was similar between the two groups (Table 1). The average length of stay was 1.6 days in the SLIP group and 2.1 in the control group $(p=0.42)$. On average, pain control required $34 \mathrm{mg}$ of morphine equivalents in the SLIP group and $26 \mathrm{mg}$ of morphine equivalents in the control group $(p=0.1793)$.

\section{Discussion}

Laparoscopic nephrectomy affords dramatic improvement in cosmesis and convalescence over open surgical nephrectomy, confirmed in several randomized clinical trials. ${ }^{12-16}$ Laparoscopic renal surgery has several variations and has seen many advances over the years since the Clayman's first case performed in $1990 .{ }^{17}$ Minimally invasive surgeons continually search for ways to improve cosmesis even more, and reduce the duration and intensity of convalescence. The most recent alternative to standard laparoscopy is LESS. Proponents of LESS have touted improved cosmesis as the major advantage of the technique, and have suggested that improvements in overall convalescence and morbidity will follow. ${ }^{4,18,19}$ The technical difficulty of LESS, however, is well recognized and impedes its use.

The impetus behind developing SLIP was a desire to rise to LESS's challenge of improving cosmesis while also maintaining the utility and versatility of multiple port sites so as to avoid the technical restrictions of LESS. The 2 main principles of SLIP are (1) use of the smallest ports that can accommodate the required instruments, and (2) strategic use of existing anatomical features (umbilicus, pubic hairline, scars, body art, and body piercings) to hide the incisions. The results of the 


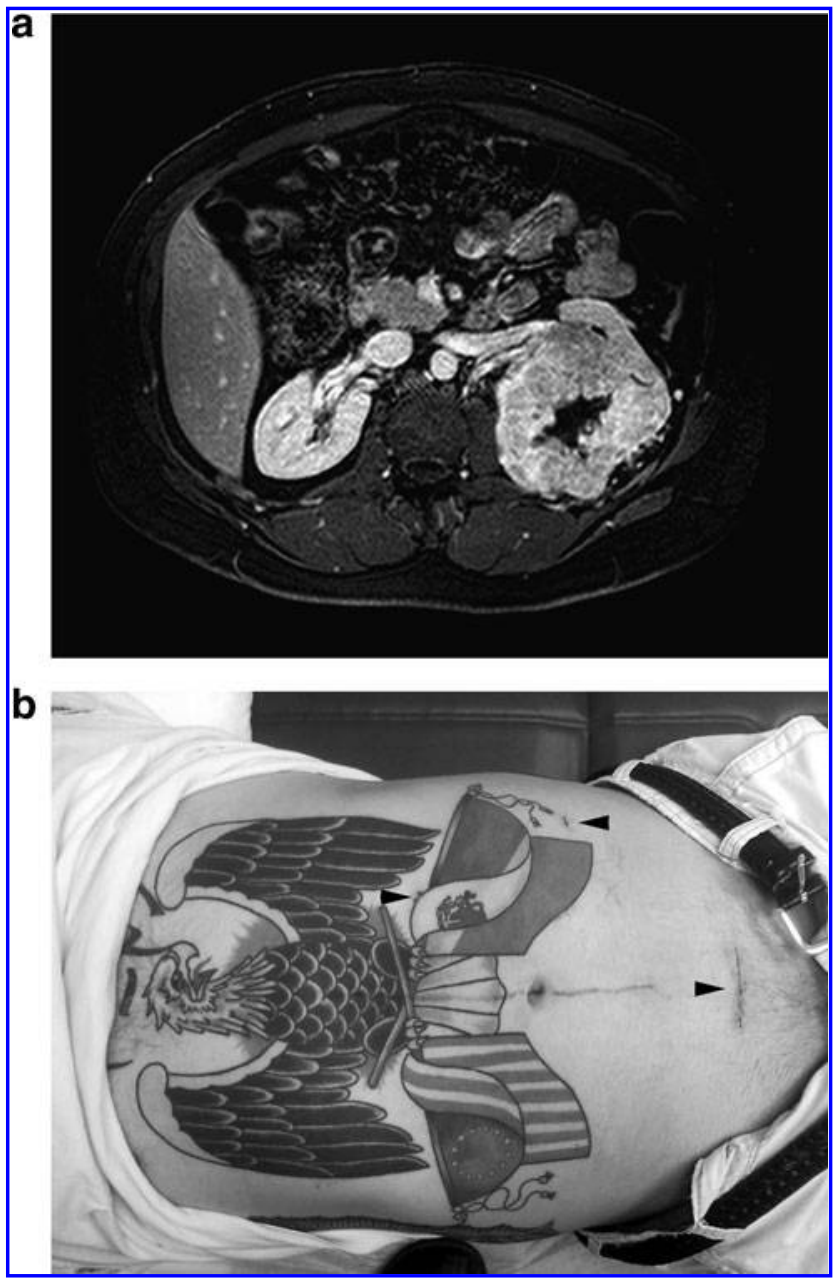

FIG. 3. (a) MRI of large left renal mass. (b) Abdominal scars in the same patient (body mass index $=36.6 \mathrm{~kg} / \mathrm{m}^{2}$ ) 2 weeks after SLIP radical nephrectomy. The incision for the 5 -mm umbilical port (laparoscope) is not visible. The incisions for the $4 \mathrm{~cm}$ suprapubic extraction site and the two 5-mm working ports are marked with black arrowheads.

current study, and the expansion of our SLIP principles to additional procedures, suggest that SLIP is a favorable alternative to LESS.

In our series, the patient BMI and age range was vast, and did not affect the choice to proceed with the SLIP nephrectomy. Of the SLIP patients, 24\% were obese (BMI $30-40 \mathrm{~kg} / \mathrm{m}^{2}$ ) (Fig. 3) and 10\% were morbidly obese (BMI $>40 \mathrm{~kg} / \mathrm{m}^{2}$ ), with a mean BMI of $29.4 \mathrm{~kg} / \mathrm{m}^{2}$. In three recent publications comparing LESS to standard laparoscopic procedures in which detailed BMI information was provided, the average BMI in the LESS patients was $27 \mathrm{~kg} / \mathrm{m}^{2}$ and very few patients had $\mathrm{BMI}>30 \mathrm{~kg} / \mathrm{m}^{2}$. 3,4,6 Additionally, trainees were the primary surgeons in the majority of our SLIP procedures, and there were no significant differences in intraoperative and postoperative measures aside from a statistically significant but clinically insignificant 23 minutes longer mean operative time in the SLIP group, further supporting the contention that the SLIP nephrectomy is not significantly more technically difficult than a standard laparoscopic nephrectomy. In comparison, when reviewing the literature on LESS it appears that

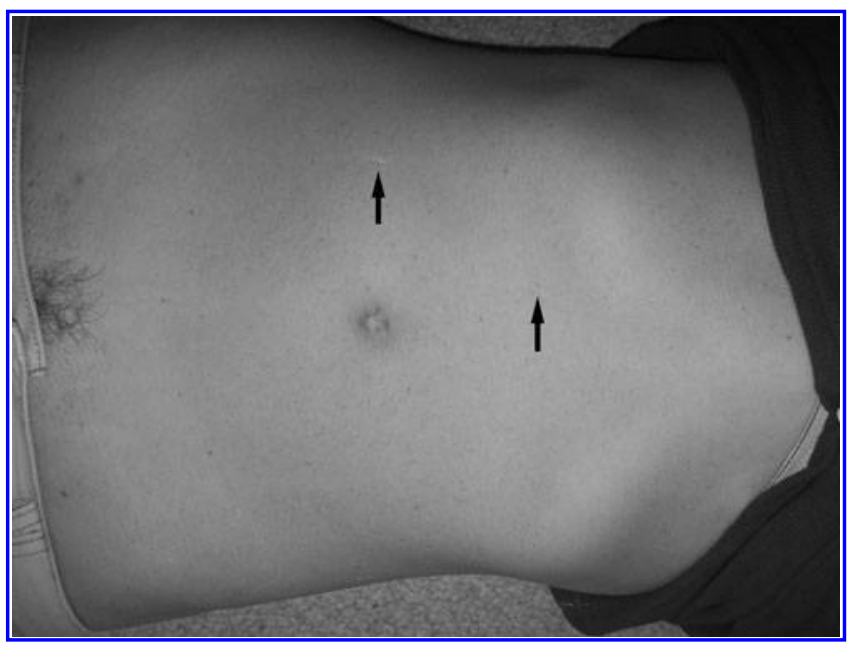

FIG. 4. Abdominal scars 6 months after right SLIP heminephrectomy. The incisions for the 5-mm umbilical port (laparoscope) and the 12-mm suprapubic port (assisting port and extraction site) are not visible. The incisions for the working 5-mm ports (black arrows) are barely discernible.

these procedures were performed in ideal patient populations with extensively trained attending urologists as the primary surgeons, given the skill level needed to operate through a single incision. ${ }^{7,19-22}$ The technical challenges of LESS renal surgery are stressed repeatedly in the literature.

From a cosmetic standpoint, LESS does use only one incision site, making for improved cosmesis compared with standard laparoscopy. However, our experience is that small and strategically placed ports, taking advantage of the base of the umbilicus and placement of the only incision $>5 \mathrm{~mm}$ below the pubic hairline (and other anatomical features), will also improve the cosmetic outcome (Fig. 4). In fact, it might be argued that the cosmetic result with SLIP (only two visible $5 \mathrm{~mm}$ incisions) might be superior to that of LESS. Although incision length was comparable between our two groups, the cosmetic benefit of the SLIP approach owes to the differences in port positioning. No incisions $>5 \mathrm{~mm}$ from the SLIP group were placed outside the umbilicus, suprapubic, or lower midline positions. Although we and our patients are pleased with the cosmetic outcome, we have not compared cosmesis with that of standard laparoscopy (or with LESS) using a validated objective instrument, which is a limitation to this study.

Despite smaller incisions or even a solitary incision, it appears that convalescence differs insignificantly between standard laparoscopy, LESS, and our SLIP technique. Clearly, this is an outcome of great interest that could give advantage to one approach over the others. The preponderance of literature comparing standard laparoscopy and LESS for renal surgery published to date suggests that there is no difference between the two approaches in terms of postoperative convalescence. Of nine comparisons of LESS and standard laparoscopic simple/radical/donor nephrectomy, pyeloplasty, cryo-ablation, or adrenalectomy published to date, ${ }^{2-10}$ only three reported lower visual analog pain scores in the LESS patients, $4,8,10$ only two found less postoperative narcotic use in the LESS patients, ${ }^{5,8}$ and in only one was the duration of hospital stay shorter in the LESS patients. ${ }^{10}$ It appears likely 

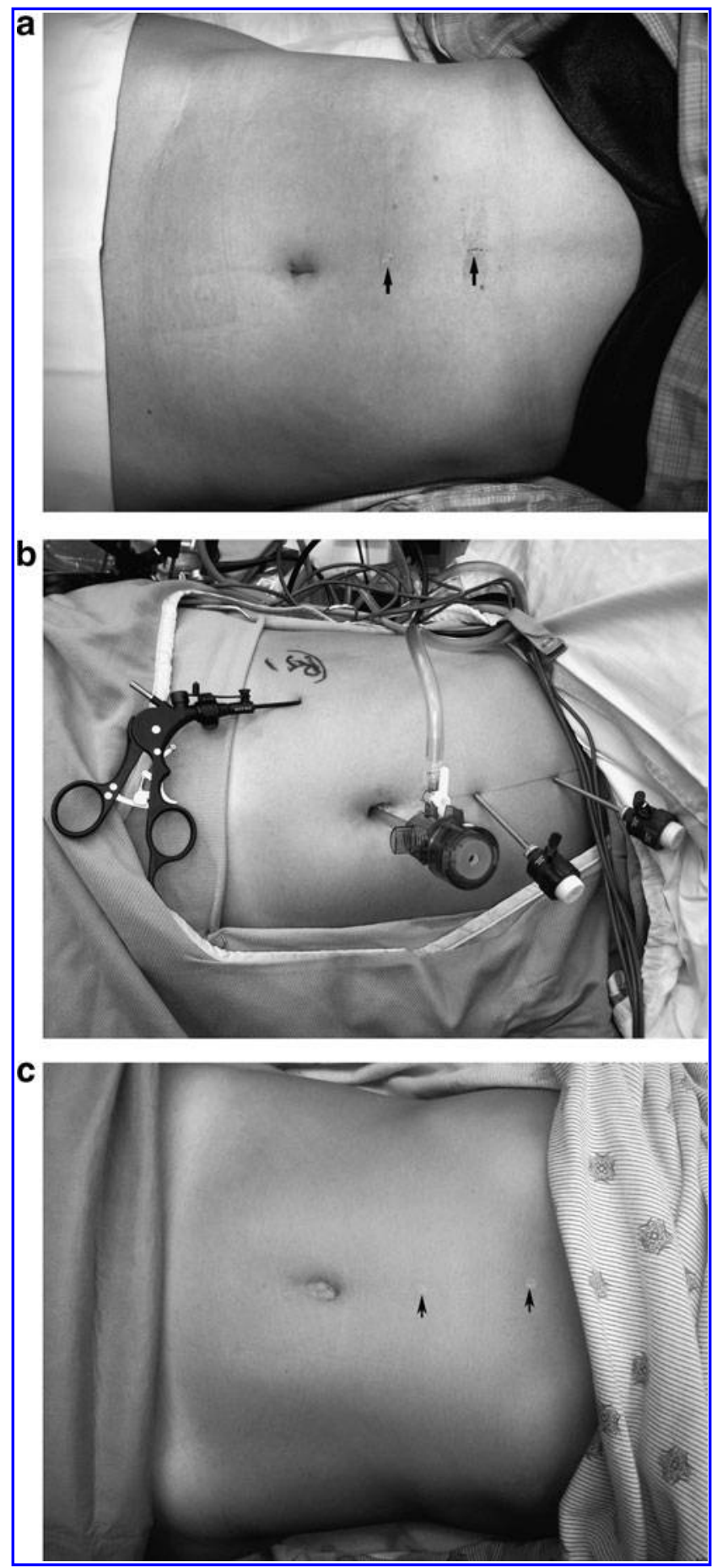

FIG. 5. (a) Abdominal scars 2 weeks after right pyeloplasty using a 12-mm port in the umbilicus (also used for the postoperative drain), two $5-\mathrm{mm}$ ports in the midline above the umbilicus (black arrows), and a 2-mm grasper placed through a small incision that is not visible. (b) Port placement for right pyeloplasty using a 5-mm port in the umbilicus (also used for the postoperative drain), two 3-mm ports in the midline above the umbilicus, and a 2-mm grasper placed through a small incision without a port. (c) Abdominal scars 2 weeks after the procedure in (b). The scars from the $3-\mathrm{mm}$ ports are less noticeable than the ones from the $5-\mathrm{mm}$ ports (black arrows) and the $2 \mathrm{~mm}$ incision is not visible.

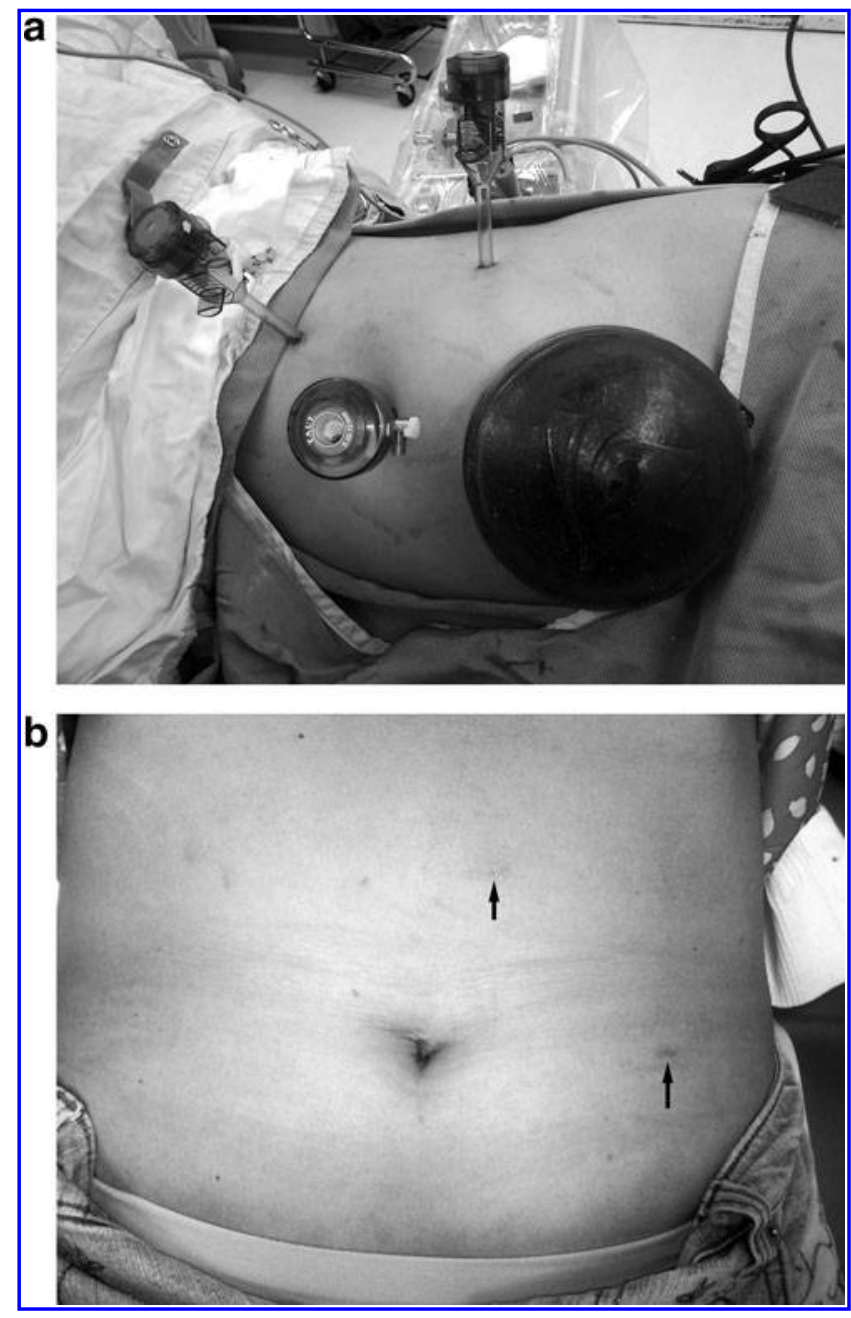

FIG. 6. (a) Port placement for hand-assisted laparoscopic left donor nephrectomy, with the hand-assistance device placed through an 11-cm suprapubic transverse incision (the longer incision allowed for the fascial incision to be moved cephalad to an intraperitoneal location), a 12-mm port in the base of the umbilicus, and two 5-mm ports. (b) Two weeks later, only the two 5-mm incisions (black arrows) are visible above the undergarments.

that, once incision size is reduced to a certain level, the postoperative pain owing to the internal manipulation becomes predominant and further reductions in incision size bring limited if any improvement in convalescence.

Although LESS is certainly receiving attention for ingenuity, its only clear advantages to date in urologic renal surgery have been limited to improved cosmetic outcome. ${ }^{21}$ This improvement comes with technical challenges and a steep learning curve, even in very selected patient populations. By using small ports that are strategically placed, we are able to maintain the standard and well-known approach to laparoscopic renal surgery while also improving the cosmetic results. We have modified our approach to laparoscopic pyeloplasty and selected partial and donor nephrectomies using the principles of SLIP. For pyeloplasties and selected partial nephrectomies, the only 12-mm port (for needles and larger instruments) is placed in the base of the umbilicus, and this site is the one used 
for the postoperative drain. A small incision can be made for a 2-mm assisting grasper passed directly through the skin without a port, and this wound closes without sutures. The only incisions visible without close inspection are for two or three 5-mm port sites (Fig. 5a). We recently have started using 3-mm ports (needlescopic laparoscopy, or microlaparoscopy) for smaller working instruments in pyeloplasties. One 5- or 12$\mathrm{mm}$ port (depending on the suturing technique) is placed in the base of the umbilicus, two 3-mm ports are placed for working instruments, and a 2-mm grasper is placed directly through the skin without a port (Fig. 5b). The cosmetic result is truly outstanding, with only barely discernible scars only 3 weeks later (Fig. 5c). In the case of donor nephrectomy, a procedure for which we still prefer hand-assistance, in selected cases the hand-assistance device can be placed in a transverse suprapubic site below the pubic hairline, the only $12-\mathrm{mm}$ port is placed in the base of the umbilicus, and the only incisions visible without close inspection are for two additional 5-mm ports (Fig. 6).

In conclusion, many laparoscopic renal procedures can be performed using two or three 5 -mm ports in visible locations, a 5 - or 12-mm port (+/-drain site) in the base of the umbilicus, and any larger incision in a transverse suprapubic site below the pubic hairline. This approach provides excellent cosmetic results, without the limitations of LESS. Use of microlaparoscopic instruments and ports adds to the reduced cosmetic impact. Given the ease of the SLIP technique and its relevance to all surgeons while using standard multi-port laparoscopic techniques, the SLIP technique should be considered an alternative to LESS when improved cosmesis is desired.

\section{Disclosure Statement}

No competing financial interests exist.

\section{References}

1. Ponsky LE, Steinway ML, Lengu IJ, et al. A Pfannenstiel single-site nephrectomy and nephroureterectomy: A practical application of laparoendoscopic single-site surgery. J Urol 2009;74:482-485.

2. Raman JD, Bagrodia A, Cadeddu JA. Single-incision, umbilical laparoscopic versus conventional laparoscopic nephrectomy: A comparison of perioperative outcomes and short-term measures of convalescence. Eur Urol 2009;55:1198-1206.

3. Tracy CR, Raman JD, Bagrodia A, et al. Perioperative outcomes in patients undergoing conventional laparoscopic versus laparoendoscopic single-site pyeloplasty. Urology 2009;74:1029-1034.

4. White WM, Goel RJ, Kaouk JH. Single-port laparoscopic retroperitoneal surgery: Initial operative experience and comparative outcomes. J Urol 2009;73:1279-1282.

5. Jeong BC, Park JH, Han DH, et al. Laparoendoscopic singlesite and conventional laparoscopic adrenalectomy: A matched case-control study. J Endourol 2009;23:1957-1960.

6. Andonian S, Rais-Bahrami S, Atalla MA, et al. Laparoendoscopic single-site pfannenstiel versus standard laparoscopic donor nephrectomy. J Endourol 2010;24:429-432.

7. Raybourn JH, III, Rané A, Sundaram CP. Laparoendoscopic single-site surgery for nephrectomy as a feasible alternative to traditional laparoscopy. Urology 2010;75:100-103.

8. Tugcu V, Ilbey YO, Mutlu B, et al. Laparoendoscopic singlesite surgery versus standard laparoscopic simple nephrectomy: A prospective randomized study. J Endourol 2010;24: 1315-1320.
9. Canes D, Berger A, Aron M, et al. Laparo-endoscopic single site (LESS) versus standard laparoscopic left donor nephrectomy: Matched-pair comparison. Eur Urol 2010;57:95-101.

10. Park YH, Park JH, Jeong CW, et al. Comparison of laparoendoscopic single-site radical nephrectomy with conventional laparoscopic radical nephrectomy for localized renal-cell carcinoma. J Endourol 2010;24:997-1003.

11. Gabr AH, Gdor Y, Strope SA, et al. Approach and specimen handling do not influence oncological perioperative and long-yerm outcomes after laparoscopic tadical nephrectomy. J Urol 2009;182:874-880.

12. Wolf JS, Jr., Merion RM, Leichtman AB, et al. Randomized controlled trial of hand-assisted laparoscopic versus open surgical live donor nephrectomy. Transplantation 2001;72: 284-290.

13. Simforoosh N, Basiri A, Tabibi A, et al. Comparison of laparoscopic and open donor nephrectomy: A randomized controlled trial. BJU Int 2005;95:851-855.

14. Kok NFM, Lind MY, Hansson BME, et al. Comparison of laparoscopic and mini incision open donor nephrectomy: Single blind, randomised controlled clinical trial. BMJ 2006; 333:221-226.

15. Andersen $\mathrm{MH}$, Mathisen $\mathrm{L}$, Øyen $\mathrm{O}$, et al. Postoperative pain and convalescence in living kidney donors-laparoscopic versus open donor nephrectomy: A randomized study. Am J Transplant 2006;6:1438-1443.

16. Burgess NA, Koo BC, Calvert RC, et al. Randomized trial of laparoscopic vs. open nephrectomy. J Endourol 2007;21:610613.

17. Clayman RV, Kavoussi LR, Soper NJ, et al. Laparoscopic nephrectomy: Initial case report. J Urol 1991;146:278-282.

18. Raman JD, Cadeddu JA, Rao P, et al. Single-incision laparoscopic surgery: Initial urological experience and comparison with natural-orifice transluminal endoscopic surgery. BJU Int 2008;101:1493-1496.

19. Desai MM, Berger AK, Brandina R, et al. Laparoendoscopic single site surgery: Initial hundred patients. Urology 2009; 74:805-812.

20. Stolzenburg JU, Hellawell G, Kallidonis P, et al. Laparoendoscopic single-site surgery: Early experience with tumor nephrectomy. J Endourol 2009;23:1287-1292.

21. Irwin BH, Rao PP, Stein RJ, et al. Laparoendoscopic single site surgery in Urology. Urol Clin North Am 2009;36: 223-235.

22. Rane A, Ahmed S, Kommu SS, et al. Single-port "scarless" laparoscopic nephrectomies: The United Kingdom experience. BJU Int 2009;104:230-233.

Address correspondence to: J. Stuart Wolf, Jr., M.D. Department of Urology University of Michigan Health System 1500 East Medical Center Drive, TC 3875 Ann Arbor, MI 48109-5330

E-mail: wolfs@umich.edu

\section{Abbreviations Used}

ASA $=$ American Society of Anesthesiologists

$\mathrm{BMI}=$ body mass index

LESS = laparoendoscopic single-site surgery

LOS $=$ length of stay

$\mathrm{SLIP}=$ small strategic laparoscopic incision placement 